DOI: 10.20472/IAC.2017.029.027

\author{
WITOLD ORZESZKO \\ Nicolaus Copernicus University in Torun, Poland
}

\title{
NONPARAMETRIC PREDICTION OF NONLINEAR TIME SERIES. A MONTE CARLO STUDY
}

\begin{abstract}
:
Nonparametric regression is an alternative to the parametric approach, which consists of applying parametric models, i.e models of the certain functional form with a fixed number of parameters. As opposed to the parametric approach, nonparametric models have a general form, which can be approximated increasingly precisely when the sample size grows. Hereby they do not impose such restricted assumptions about the form of the modelling dependencies and in consequence, they are more flexible and let the data speak for themselves.

One of the most popular nonparametric regression method is kernel smoothing, dating back to Rosenblatt (1956) and Parzen (1962). Nowadays, there are a number of variations of the kernel smoothers. In the paper, the local-linear kernel regression is assessed using a Monte Carlo study. The study considered varied linear and nonlinear data generating processes, comprising chaotic systems and the well-known in econometrics stochastic processes with nonlinearity in the mean and in the variance.
\end{abstract}

\section{Keywords:}

Local-linear kernel smoother, nonparametric prediction, nonlinear time series.

JEL Classification: C53, C14 